\title{
Avaliação agronômica para seleção de genótipos de amendoim visando precocidade
}

Submetido - 06 Jul. $2021 \quad$ Aprovado - 01 out. $2021 \quad$ Publicado - 21 out. 2021

http://dx.doi.org/10.52755/sas.v.2i(edesp1)132

\begin{abstract}
Jair Heuert (iD
Programa de Melhoramento do Amendoim - Embrapa, Santo Antônio de Goiás, GO. E-mail: jair.heuert@embrapa.br.
\end{abstract}

Maxuel Fellipe Nunes Xavier (iD

Discente de Agronomia do IFMT Campus São Vicente - Centro de Referência de Campo Verde, Campo Verde, MT. E-mail: maxuelfellipe90@gmail.com.

Taís de Moraes Falleiro Suassuna (iD

Programa de Melhoramento do Amendoim - Embrapa, Santo Antônio de Goiás, GO. E-mail: tais.suassuna@embrapa.br.

\section{RESUMO}

O presente trabalho teve como objetivo avaliar as características agronômicas de genótipos de amendoim no estado de Goiás. O experimento foi realizado na área experimental da EMBRAPA Arroz e Feijão, no ano agrícola 2020/21, com semeadura manual realizada no dia 10 de dezembro de 2020. Foi utilizado delineamento em blocos casualizados, com quatro repetições. Os tratamentos constaram de oito genótipos, sendo sete linhagens (2133 OL, 2266 OL, 2300 OL, 1253 OL, 2250 OL, 2101 OL e 2717 OL) e uma cultivar (BRS 423 OL), todas desenvolvidas pelo PMA da EMBRAPA. As parcelas foram compostas por duas linhas de três metros de comprimento, espaçamento ente linhas de $0,90 \mathrm{~m}$, intervalo entre parcelas de dois metros $e$ parcela útil $5,4 \mathrm{~m}^{2}$. Os manejos cultural e fitossanitário foram realizados de acordo com as recomendações para a cultura. $O$ estande médio foi de 14 plantas por metro linear. $O$ ensaio foi invertido no dia 12 de abril de 2021, aos 123 DAP. Conclui-se que os genótipos 2133 OL, BRS $423 O L$ e 2266 OL obtiveram as menores severidades de mancha preta. Somente o $2717 \mathrm{OL}$, 2133 OL, 2101 OL e 2266 OL apresentaram maturação dos grãos acima de 70\%. Houve diferença significativa entre as massas de 100 grãos, podendo atender diferentes nichos de mercado. Os mais produtivos foram o 2717 OL, 2101 OL, 2266 OL, 2250 OL, 1253 OL e 2133 OL

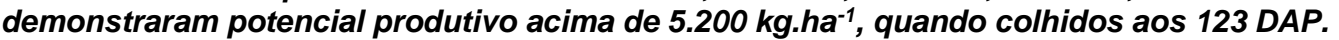

Palavras-chave: Arachis hypogaea L.; Programa de Melhoramento; Precocidade; Produtividade.

\section{Agronomic evaluation for selection of peanut genotypes aiming precocity}

\section{ABSTRACT}

This study aimed to evaluate the agronomic characteristics of peanut genotypes in the state of Goiás. The experiment was carried out in the experimental area of EMBRAPA Arroz e Feijão, in the agricultural year 2020/21, with manual sowing carried out on December 10, 2020. A randomized block design with four replications was used. The treatments consisted of eight genotypes, seven lines (2133 OL, 2266 OL, 2300 OL, 1253 OL, 2250 OL, 2101 OL and 2717 OL) and one cultivar (BRS $423 \mathrm{OL}$ ), all developed by the PMA from EMBRAPA. The plots were composed of two lines of three meters in length, spacing between lines of $0.90 \mathrm{~m}$, interval between plots of two meters and useful plot of $5.4 \mathrm{~m}^{2}$. The cultural and phytosanitary management were carried out in accordance with the recommendations for the crop.

Este é um trabalho de acesso aberto e distribuído sob os Termos da Creative Commons Attribution Attribution-NonCommercial-ShareAlike 4.0 International. 
The average stand was 14 plants per linear meter. The test was reversed on April 12, 2021, at 123 DAP. It is concluded that the genotypes $2133 \mathrm{OL}$, BRS $423 \mathrm{OL}$ and $2266 \mathrm{OL}$ had the lowest black spot severities. Only 2717 OL, 2133 OL, 2101 OL and 2266 OL showed grain maturation above $70 \%$. There was a significant difference between the masses of 100 grains, which could serve different market niches. The most productive were 2717 OL, 2101 OL, 2266 OL, 2250 OL, $1253 O L$ and 2133 OL showed productive potential above 5,200 kg.ha-1, when harvested at 123 DAP.

Keywords: Arachis hypogaea L.; Improvement Program; Precocity; Productivity.

\section{Evaluación agronómica para la selección de genotipos de} maní con el objetivo de precocidad

\section{RESUMEN}

Este estudio tuvo como objetivo evaluar las características agronómicas de genotipos de maní en el estado de Goiás. El experimento se realizó en el área experimental de EMBRAPA Arroz e Feijão, en el año agrícola 2020/21, con siembra manual realizada el 10 de diciembre 2020 Se utilizó un diseño de bloques al azar con cuatro repeticiones. Los tratamientos consistieron en ocho genotipos, siete líneas (2133 OL, 2266 OL, 2300 OL, 1253 OL, 2250 OL, 2101 OL y 2717 OL) y un cultivar (BRS 423 OL), todos desarrollados por la PMA de la EMBRAPA. Las parcelas estaban compuestas por dos líneas de tres metros de longitud, espaciamiento entre líneas de $0,90 \mathrm{~m}$, intervalo entre parcelas de dos metros y parcela útil de $5,4 \mathrm{~m}^{2}$. El manejo cultural y fitosanitario se realizó de acuerdo con las recomendaciones para el cultivo. El rodal promedio fue de 14 plantas por metro lineal. La prueba se revirtió el 12 de abril de 2021 a 123 DAP. Se concluye que los genotipos 2133 OL, BRS 423 OL y 2266 OL tuvieron las menores severidades de mancha negra. Solo 2717 OL, 2133 OL, 2101 OL y 2266 OL mostraron una maduración de grano superior al 70\%. Hubo una diferencia significativa entre las masas de 100 granos, que podrían servir a diferentes nichos de mercado. Los más productivos fueron 2717 OL, 2101 OL, 2266 OL, 2250 OL, 1253 OL y 2133 OL mostraron un potencial productivo superior a 5.200 kg.ha1 , cuando se cosecharon a 123 DAP.

Palabras clave: Arachis hypogaea L.; Programa de Mejoramiento; Maní de ciclo curto; Productividad.

\section{Introdução}

No Brasil, o cultivo do amendoim concentra-se na região de São Paulo, onde ocupa uma área de 150,0 mil hectares, correspondendo a mais de $90 \%$ da área plantada no Brasil (159,7 mil hectares), representando um crescimento de 4,2\% em relação ao ciclo anterior (143,9 mil hectares). O volume produzido foi de 555,0 mil toneladas, um aumento de $7,9 \%$ em relação à safra anterior (514,2 mil toneladas) (CONAB, 2021).

Os agricultores cultivam amendoim em áreas para reforma de canavial. Nesse sistema de produção, a safra da cana-de-açúcar é mantida por aproximadamente 5 anos e quando os níveis de produção diminuem, recomenda-se a inserção de uma leguminosa para que ocorra a rotação de culturas e posterior retorno do canavial (MACEDO; SEABRA, 2008). 
Nesse cenário, após a colheita do amendoim, tem-se um curto período para o plantio da cana-de-açúcar, devido à diminuição das chuvas de verão. Portanto, é fundamental a utilização de cultivares de amendoim que apresentem um elevado grau de precocidade para otimizar esse processo (BETIOL et al., 2019). Por outro lado, a soja conquistou a preferência de produtores e usinas de cana-de-açúcar nessas regiões, onde tradicionalmente cultivavam o amendoim. Pois a soja tem sido mais atrativa devido ao ciclo que varia de 105 a 120 dias e principalmente pelo estabelecimento de um novo plantio de cana-de-açúcar, enquanto o ciclo das cultivares de amendoim mais plantadas variam de 130 a 150 dias.

Segundo os dados do Observatório da Cana (2021), a cada ano uma área de 850 mil hectares de cana-de-açúcar é disponibilizada para reforma apenas na região de São Paulo. Nesse sentido, outra região promissora é o Triângulo Mineiro, em Minas Gerais, que possui uma grande área de canade-açúcar e cada ano contribui com uma área de rotação superior a 120 mil hectares. Uma grande indústria de processamento de amendoim e óleo está sendo instalada em Iturama-MG, o que permitirá a expansão do cultivo do amendoim para novas regiões.

O Brasil, tem enorme potencial para aumentar significativamente sua produção e ser o maior exportador mundial de amendoim, porém esse crescimento está diretamente ligado à pesquisa e ao desenvolvimento de novas cultivares de ciclo precoce, que são a base para a sustentabilidade de toda a produção (VIZEU, 2019). Assim, para atender a essa demanda, o Programa de Melhoramento do Amendoim (PMA) da Empresa Brasileira de Pesquisa Agropecuária (EMBRAPA), possui diversas parcerias públicas e privadas e vem intensificando suas pesquisas para obtenção de novas cultivares de ciclo precoce. Além do ciclo desejado, o melhoramento genético busca selecionar cultivares que atendam às demandas dos produtores, proporcionando alta produtividade, resistência às principais pragas e doenças, alto teor de ácido oleico, tamanho e formato do grão, além de visual, nutricional, químico e sensorial. 
Com as novas combinações que utilizam acessos a bancos de germoplasma, principalmente do Nordeste, foi possível reduzir o ciclo. Os estudos na última safra, de Martins et al. (2020), foram realizados com a colheita aos 125 dias após o plantio (DAP), utilizando as novas linhagens. A avaliação utilizada foi a do grau de maturação do grão como componente determinante do ciclo de uma cultivar, realizada visualmente de acordo com o grau de enchimento do grão, com a finalidade de selecioná-lo de acordo com a maturação precoce. Com isso, observaram-se que as linhagens 2173 OL, $2250 \mathrm{OL}$ e $2133 \mathrm{OL}$ obtiveram os maiores percentuais de grãos maduros, configurando-se como de ciclo precoce naquelas condições de estudo. No entanto, podem ser promissoras e futuras cultivares para recomendação de plantio, visando uma colheita antecipada, ou seja, de ciclo precoce para atender as áreas de renovação dos canaviais. Com base nisso, o presente trabalho teve como objetivo avaliar as características agronômicas de genótipos de amendoim no estado de Goiás, visando a precocidade.

\section{Material e métodos}

O experimento foi realizado na área experimental da EMBRAPA Arroz e Feijão, na latitude de $16^{\circ} 28^{\prime} 00^{\prime \prime}$ (S), longitude de 49 17'00" (W) e altitude de $823 \mathrm{~m}$. O solo é caracterizado como Latossolo Vermelho-Escuro, de textura argilosa e relevo plano. A precipitação pluvial média anual é de $1.472,8 \mathrm{~mm}$ e umidade relativa média do ar anual é de $70 \%$. A temperatura média do ar anual é de $23,1{ }^{\circ} \mathrm{C}$, apresentando período chuvoso de outubro a abril e período seco de maio a setembro (SILVA et al., 2000). O clima é do tipo Aw, clima tropical com estação seca de inverno (KÖPPEN; GEIGER, 1928).

A semeadura foi realizada manualmente no dia 10 de dezembro de 2020. Foi utilizado delineamento em blocos casualizados, com quatro repetições. Os tratamentos foram oito genótipos, sendo sete linhagens (2133 OL, 2266 OL, 2300 OL, 1253 OL, 2250 OL, 2101 OL e 2717 OL) e uma cultivar (BRS $423 \mathrm{OL}$ ), todas desenvolvidas pelo PMA da Embrapa. 
As parcelas foram compostas por duas linhas de três metros de comprimento, espaçamento ente linhas de 0,90 m, intervalo entre parcelas de dois metros e parcela útil $5,4 \mathrm{~m}^{2}$. Os manejos cultural e fitossanitário foram realizados de acordo com as recomendações para a cultura. $\mathrm{O}$ estande médio foi de 14 plantas por metro linear. $O$ ensaio foi invertido no dia 12 de abril de 2021, aos 123 DAP.

Com base na análise química do solo da área experimental foi realizada uma adubação de semeadura de $200 \mathrm{~kg} \mathrm{ha}^{-1}$ de Superfosfato Simples. Em cobertura foi feita aplicação de $500 \mathrm{~kg} \cdot \mathrm{ha}^{-1}$ de gesso agrícola, aos 42 DAP. Foram avaliados grãos maduros (\%), severidade de mancha preta (notas) massa de 100 grãos (g) e produtividade de vagens $\left(\mathrm{kg} \cdot \mathrm{ha}^{-1} \mathrm{e}\right.$ sacas.alqueire $\left.{ }^{-1}\right)$. Os grãos maduros (\%) foram avaliados como descrito por Rucker et al. (1994).

A severidade de mancha preta (Cercosporidium personatum) foi avaliada usando a escala diagramática da incidência com notas de 1 a 9 ao final do ciclo dos genótipos, antes da inversão do experimento (SUBRAHMANYAM et al., 1982). Foram avaliados massa de 100 grãos (g) e produtividade de vagens $\left(\mathrm{kg}^{-h^{-1}}{ }^{-1}\right.$ e sacas.alqueire $\left.{ }^{-1}\right)$, mediante a pesagem de vagens e grãos, da área de $3,6 \mathrm{~m}^{2}$ centrais das duas linhas da parcela.

Os dados obtidos foram submetidos à análise de variância (Teste $\mathrm{F}$ ) e as médias dos tratamentos foram comparados pelo teste de Scott-Knott a $5 \%$ de probabilidade, por meio do programa computacional SISVAR 5.6 (FERREIRA, 2019).

\section{Resultados e discussão}

Houve diferença significativa para os diferentes genótipos de amendoim dentre as variáveis severidade de mancha preta (nota), grãos maduros (\%), massa de 100 grãos (g) e produtividade de vagens $\left(\mathrm{kg}^{\mathrm{ha}} \mathrm{ha}^{-1}\right)$. Inicialmente, na severidade, observa-se as maiores notas no $2717 \mathrm{OL}(6,6)$, 2101 OL $(6,6)$ e $2250 \mathrm{OL}(6,0)$. Por outro lado, as menores notas foram obtidas com o $2133 \mathrm{OL}(3,5)$, BRS $423 \mathrm{OL}(4,2)$ e $2266 \mathrm{OL}(4,3)$ (Tabela 1). 
Tabela 1. Severidade de mancha preta (nota), grãos maduros (\%), massa de 100 grãos $(\mathrm{g})$ e produtividade de vagens $\left(\mathrm{kg} \cdot \mathrm{ha}^{-1}\right.$ e sacas.alqueire $\left.{ }^{-1}\right)$ em função de genótipos de amendoim visando a colheita antecipada no estado de Goiás. Santo Antônio de Goiás-GO, 2020/21.

\begin{tabular}{|c|c|c|c|c|c|}
\hline \multirow{2}{*}{ Genótipos } & \multirow{2}{*}{$\begin{array}{c}\text { Severidade } \\
\text { (nota) }\end{array}$} & \multirow{2}{*}{$\begin{array}{c}\begin{array}{c}\text { Grãos } \\
\text { maduros }\end{array} \\
(\%)\end{array}$} & \multirow{2}{*}{$\begin{array}{c}\text { Massa de } \\
100 \text { grãos } \\
\text { (g) }\end{array}$} & \multicolumn{2}{|c|}{ Produtividade de vagens } \\
\hline & & & & $\left(\mathrm{kg} \cdot \mathrm{ha}^{-1}\right)$ & $\left(\right.$ sacas.alqueire $\left.^{-1}\right)$ \\
\hline $2717 \mathrm{OL}$ & $6,6 \mathrm{a}$ & $79,0 \mathrm{a}$ & $73,9 \mathrm{~b}$ & $5.763,1 \mathrm{a}$ & 557,9 \\
\hline $2133 \mathrm{OL}$ & $3,5 \mathrm{c}$ & $76,3 \mathrm{a}$ & $75,2 \mathrm{a}$ & $5.245,7 \mathrm{a}$ & 507,8 \\
\hline $2101 \mathrm{OL}$ & $6,6 \mathrm{a}$ & $71,8 \mathrm{~b}$ & $76,1 \mathrm{a}$ & $5.657,1 \mathrm{a}$ & 547,6 \\
\hline $2266 \mathrm{OL}$ & $4,3 \mathrm{c}$ & $70,0 \mathrm{~b}$ & $73,9 \mathrm{~b}$ & $5.474,9 \mathrm{a}$ & 530,0 \\
\hline $2250 \mathrm{OL}$ & $6,0 \mathrm{a}$ & $69,8 \mathrm{~b}$ & $75,3 \mathrm{a}$ & $5.273,4 \mathrm{a}$ & 510,5 \\
\hline $1253 \mathrm{OL}$ & $5,4 \mathrm{~b}$ & $66,8 \mathrm{~b}$ & $73,9 \mathrm{~b}$ & $5.267,2 \mathrm{a}$ & 509,8 \\
\hline BRS $423 \mathrm{OL}$ & $4,2 \mathrm{c}$ & $61,8 \mathrm{c}$ & $75,6 \mathrm{a}$ & $4.328,9 \mathrm{~b}$ & 419,0 \\
\hline $2300 \mathrm{OL}$ & $5,1 \mathrm{~b}$ & $56,8 d$ & $73,6 \mathrm{~b}$ & $4.756,9 \mathrm{~b}$ & 460,5 \\
\hline Média & 5,2 & 69,0 & 74,7 & $5.220,9$ & 505,4 \\
\hline C.V. (\%) & 9,8 & 4,9 & 0,9 & 8,3 & - \\
\hline $\mathrm{Pr}>\mathrm{Fc}$ & $>0,0001^{*}$ & $>0,0001^{*}$ & $0,0001^{*}$ & $0,0018^{*}$ & - \\
\hline
\end{tabular}

Corroborando com Martins et al. (2020), que obtiveram efeito significativo com os diferentes genótipos do PMA testados, com maior nota no $2101 \mathrm{OL}(7,3)$ e menores na $2250 \mathrm{OL}(5,3), 2133 \mathrm{OL}(5,3), 2266 \mathrm{OL}(5,3)$ e 1253 OL $(5,8)$, demonstrando possível semelhança entre os resultados, pois ambos os experimentos foram desenvolvidos na área experimental do PMA da Embrapa Arroz e Feijão, em Santo Antônio de Goiás-GO.

Nos grãos maduros (\%), verifica-se que os genótipos 2717 OL $(79,0 \%)$ e 2133 OL $(76,3 \%)$ apresentaram as maiores porcentagens de grãos maduros. De modo intermediário, encontram-se o $2101 \mathrm{OL}(71,8 \%)$, 2266 OL (70,0\%), 2250 OL $(69,8 \%)$ e 1253 OL (66,8\%) (Tabela 1). No contexto de maturação, o 2717 OL, 2133 OL, 2101 OL e 2266 OL obtiveram maturação dos grãos acima de $70 \%$. Com isso, restam-se o $2300 \mathrm{OL}$ $(56,8 \%)$ e o BRS $423 \mathrm{OL}(61,8 \%)$ com as menores porcentagens.

As maiores massas de 100 grãos foram obtidas nos genótipos 2101 OL (76,1 g), BRS $423 \mathrm{OL}(75,6 \mathrm{~g}), 2250 \mathrm{OL}(75,3 \mathrm{~g})$ e $2133 \mathrm{OL}(75,2 \mathrm{~g})$. Ao contrário, tem-se o 2300 OL (73,6 g), 1253 OL (73,9 g), 2266 OL (73,9 g) e $2717 \mathrm{OL}(73,9 \mathrm{~g})$ (Tabela 1). Com base nisso, concorda-se com Martins et 
al. (2020), que obtiveram dados correlatos com os genótipos 2101 OL (74,4 g), 2250 OL (73,7 g) e BRS 423 OL (76,8 g). Ademais, Betiol et al. (2019), observaram massa de 79,6 g com o BRS $423 \mathrm{OL}$, invertido aos 127 DAP, em Ribeirão Bonito-SP.

Na produtividade de vagens, verifica-se que os genótipos 2717 OL (5.763,1 kg.ha-1), 2101 OL (5.657,1 kg.ha-1), 2266 OL (5.474,9 kg.ha-1), 2250 OL (5.273,4 kg.ha-1), 1253 OL (5.267,2 kg.ha-1) e 2133 OL (5.245,7 kg.ha-1), foram os mais produtivos do presente estudo quando comparado aos demais genótipos, obtendo produtividades superiores a 5.200 kg.ha-1 ou 503 sacas.alqueire ${ }^{-1}$ (Tabela 1).

Ao passo que o $2230 \mathrm{OL}\left(4.756,9 \mathrm{~kg} \cdot \mathrm{ha}^{-1}\right)$ e BRS $423 \mathrm{OL}(4.328,9$ $\mathrm{kg} \cdot \mathrm{ha}^{-1}$ ) apresentaram as menores produtividades (Tabela 1), configurando que ainda não estavam aptos para a inversão, já que foram os genótipos que possuem a maior porcentagem de grãos imaturos, 56,8 e 61,8 respectivamente. Considerando que o genótipo $2133 \mathrm{OL}$ esteve entre as mais produtivas tanto no presente trabalho quanto no estudo de Martins et al. (2020).

\section{Conclusões}

Conclui-se que os genótipos 2133 OL, BRS 423 OL e 2266 OL obtiveram as menores severidades de mancha preta. Somente o $2717 \mathrm{OL}$, 2133 OL, 2101 OL e 2266 OL apresentaram maturação dos grãos acima de $70 \%$. Houve diferença significativa entre as massas de 100 grãos, podendo atender diferentes nichos de mercado. Os mais produtivos foram o $2717 \mathrm{OL}$, 2101 OL, 2266 OL, 2250 OL, 1253 OL e 2133 OL demonstraram potencial produtivo acima de $5.200 \mathrm{~kg} \mathrm{ha}^{-1}$, quando colhidos aos 123 DAP.

\section{Agradecimentos}

Estes resultados estão vinculados ao projeto SEG 20.18.01.021.00. Os autores agradecem as empresas que são parceiras do Programa de Melhoramento do Amendoim - Embrapa: Agroindustrial Santo Expedito, Agro Polegatto, Agulhon Agroindustrial, Amenco Peanuts, Amendoperes, 
Amendofante, Balsamo Peanuts Company, Betiol Peanuts, Casul Peanuts, Copercana, Cerealista Fazenda Canaã, Comari Peanuts, Francfort Trade, HB Zanchetta, JM Zanchetta, LC Sementes, MGV Agroindustrial, NP Zanchetta, Pinto Neto Importação, Sementes Esperança e Sementes Manduca. Na categoria de apoio ao trabalho de pesquisa, as empresas Bokada Alimentos LTDA, JLA Brasil, Semecat, MIAC - Industrias Colombo, Tecnofuertes, Peanuts Brazil e as instituições de ensino IFMT Campus Sorriso, IFMT Campus São Vicente, UFTM e UEMS. 


\section{Referências}

BETIOL, R. A. B.; HEUERT, J.; MARTINS, K. B. B.; SUASSUNA, T. M. F. Desempenho de cultivares de amendoim tipo runner na região central do estado São Paulo, visando a colheita antecipada. In: Anais do encontro sobre a cultura do amendoim, 16., 2019, Jaboticabal. Anais eletrônicos... Campinas, GALOÁ, 2019.

CONAB. Acompanhamento da Safra Brasileira de Grãos. Safra 2020/21 - Nono levantamento, v. 8, n. 9, p. 1-121, 2021. Disponível em: $<$ https://www.conab.gov.br/info-agro/safras $>$. Acesso em: 27 jun. 2021.

FERREIRA, D. F. SISVAR: um sistema de análise de computador para efeitos fixos projetos de tipo de partida dividida. Revista Brasileira de Biometria, v. 37, n. 4, p. 529-535, 2019.

KÖPPEN, W.; GEIGER, R. Klimate der Erde. Gotha: Verlagcondicionadas. Justus Perthes. 1928.

MACEDO, I. C., SEABRA, J. E. Mitigation of GHG emissions using sugarcane bioethanol. In: ZUUBIER, P.; VOOREN, J. V. Sugarcane Ethanol: Contributions to climate change mitigation and the environment. Wageningen: Wageningen Academic Publishers. 2008. p. 95-111.

MARTINS, K. B. B.; HEUERT, J.; RODRIGUES, L. L.; XAVIER, M. F. N.; SUASSUNA, T. M. F. Desempenho agronômico de genótipos de amendoim tipo runner, visando colheita antecipada. South American Sciences, v. 1, n. 1, p. e2007, 2020.

MARTINS, K. B. B.; SUASSUNA, T. M. F.; HEUERT, J.; RIBEIRO, R. P.; SOAVE, J. H.; SANTOS, L. C. C. Avaliação em genótipos de amendoim tipo runner visando colheita antecipada. In: Anais do encontro sobre a cultura do amendoim, 15., 2018, Jaboticabal. Anais eletrônicos... Campinas, GALOÁ, 2018.

OBSERVATÓRIO DA CANA. Área cultivada com cana-de-açúcar: Mapeamento de área Centro-sul. 2021. Disponível em: $<$ https://observatoriodacana.com.br/historico-de-areainep.php?idMn=34\&tipoHistorico=6 >. Acesso em: 05 jul. 2021.

RUCKER, K. S.; KVIEN, C. C. K.; VELLIDIS, G.; HILL, N. S.; SHARPE, J. $\mathrm{K}$. A visual method of determining maturity of shelled peanuts. Peanut Science, v. 21, n. 2, p. 143-146, 1994.

SILVA, S. C.; XAVIER, L. S.; PELEGRINI, J. C.; DAVID, F. A. Informações meteorológicas para pesquisa e planejamento agrícola 1999. Santo Antônio de Goiás: EMBRAPA Arroz e Feijão, 2000. 30 p. 
SUBRAHMANYAM, P.; MCDONALD, D.; GIBBONS, R. W.; NIGAM, S. N.; NEVILL, D. J. Resistance to rust and late leaf spot diseases in some genotypes of Arachis hypogaea. Peanut Science, v. 9, p. 9-14, 1982.

VIZEU, L. A. Revista Canavieiros - Panorama da cadeia produtiva do amendoim. n. 159, p. 12-13, set. 2019. Disponível em: $<$ https://www.revistacanavieiros.com.br/uploads/pagina/tag/2019/11/e8IG HvhbxTeXEgc9/159-set-baixa.pdf>. Acesso em: 05 jul. 2021. 